Philosophical Magazine B, 1998, Vol. 77, No. 1, 49-65

\title{
Cobalt valence and crystal structure of $\mathrm{La}_{0.5} \mathrm{Sr}_{0.5} \mathrm{CoO}_{2 \cdot 25}$
}

\author{
By Z. L. WANG $\dagger$ and J. S. YIN \\ School of Materials Science and Engineering, Georgia Institute of Technology, \\ Atlanta, Georgia 30332-0245, USA
}

[Received 10 March 1997 and accepted 30 May 1997]

\begin{abstract}
Oxygen vacancies play a key role in determining the functionality and performance of many oxide smart materials. In this paper, the crystal structure of an anion-deficient perovskite-type orthorhombic (with $a \approx b$ ) phase $\mathrm{La}_{0.5} \mathrm{Sr}_{0.5} \mathrm{CoO}_{2.25}$ (or $\mathrm{La}_{8} \mathrm{Sr}_{8} \mathrm{Co}_{16} \mathrm{O}_{36}$ ) has been determined for the first time with the combined use of energy-dispersive X-ray spectroscopy, electronenergy-loss spectroscopy, high-resolution transmission electron microscopy and electron diffraction. The unit cell is made of two types of fundamental module, and it is composed of a total of eight modules. Each module is a $c$-axis stacking of the anion-deficient $\mathrm{SrCoO}_{3-z}$ and $\mathrm{LaCoO}_{3-\delta}$ basic perovskite cells. The unit cell preserves the characteristics of the perovskite framework, and it is a superstructure induced by $\mathrm{O}$ vacancies. This study illustrates the correlation of anion deficiency with the valence state of $\mathrm{Co}$, and it also proves that the $\mathrm{O}$ atom positions can be determined using the combination of transmission electron microscopy and associated techniques. This is likely to be an effective method for probing the microstructure of perovskite-type functional materials.
\end{abstract}

\section{$\S 1$. Introduction}

$(\mathrm{La}, \mathrm{A}) \mathrm{MnO}_{3}$ and $(\mathrm{La}, \mathrm{A}) \mathrm{CoO}_{3}(\mathrm{~A}=\mathrm{Ca}, \mathrm{Sr}$ or $\mathrm{Ba})$ are important materials that have many potential applications in smart systems (Prinz 1995, Wang and Kang 1997). (La, A) $\mathrm{MnO}_{3}$ type of compounds have been found to exhibit the colossal magnetoresistance (CMR) effect (Zhang et al. 1992, Chahara et al. 1993, Jin et al. 1994, Ju et al. 1994, Von Helmolt et al. 1994, Li et al. 1995) while the CMR effect of ( $\mathrm{La}, \mathrm{A}) \mathrm{CoO}_{3}$ is dramatically small (Briceno et al. 1995), although Co and Mn have similar electronic structures and valence states. It appears that full understanding of the mechanism of the CMR effect is desperately needed for optimizing and improving the performance of these materials. There are many factors correlated with the CMR ratio, one of which is the crystal structure because the CMR ratio is believed to be determined by the magnetic coupling between the intrinsic Co (or Mn) layers. The mixed valences of transition and rare-earth metals, as a typical character of functional materials, can stabilize a variety of compounds with $\mathrm{O}$ anion deficiency (Senaris- Rodriguez and Goodenough 1995). However, analysis of O vacancies is a challenge to existing microscopy techniques and it is even more difficult to thin films, because of the influence from the substrate surface, interface mismatch dislocations and defects in the film.

In the mixed-valence Co compounds, for an ordered structure with known cation doping such as $\mathrm{La}_{1-} \mathrm{Sr}_{x} \mathrm{CoO}_{3-y}$ (LSCO), the Odeficiency is directly correlated to the fraction of the mixed valence. Therefore a measurement of the Co valence state can be

$\dagger$ Author for correspondence, e-mail: zhong.wang@mse.gatech.edu. 
used to calculate the anion deficiency in the compound. This is a new approach for studying functional materials with mixed valences (Wang et al. 1997). In this paper, the structure of an anion-deficient phase $\mathrm{La}_{0.5} \mathrm{Sr}_{0.5} \mathrm{CoO}_{2.25}$ (or equivalently $\mathrm{La}_{8} \mathrm{Sr}_{8} \mathrm{Co}_{16} \mathrm{O}_{36}$ ), grown by metallic-organic chemical vapour deposition (MOCVD), is determined for the first time with the use of energy-dispersive X-ray spectroscopy (EDS), electron-energy-loss spectroscopy (EELS) and high-resolution transmission electron microscopy (HRTEM). The chemical composition is determined first using nanoprobe analysis techniques in transmission electron microscopy (TEM) (§3.1). Then the average Co valence state is measured experimentally using EELS, from which the anion deficiency is calculated (\$3.2). Based on the structural information provided by HRTEM and electron diffraction (\$3.3), an anion-deficient structural model is proposed. Calculations based on this model fit all the current available experimental data $(\S 3.4)$.

\section{§ 2. Ex PERIMENTAL Method}

The LSCO films were grown on polished $\mathrm{MgO}(001)$ substrates by the liquidsource MOCVD technique (Zhang, Gardiner, Kirlin, Boerstler and Steinbeck, 1992; Zhang, Cui, Gordon, Van Buskirs and Steinbeck, 1993). The requisite $\beta$-diketonate complexes $\mathrm{M}(\mathrm{thd})_{n}$ (where thd =2,2,6,6,-tetramethyl-3,5-heptanedionate and $\mathrm{M}=\mathrm{La}, \mathrm{Sr}$ or $\mathrm{Ca}$ ) were dissolved in an organic solvent and the solution was injected by a liquid pump into a heated stainless steel vaporizer which was maintained at $220^{\circ} \mathrm{C}$ during deposition. The film composition was controlled by varying the molar ratio of the organometallic complexes in the solution. The precursor vapour was carried upstream of the reactor inlet by nitrogen carrier gas (flow rate, $50-100 \mathrm{sccm}$ ) and the vaporization process took place on a continuous basis. The reactor pressure was maintained at 1.5-2.0 Torr. The deposition temperature was controlled at 600 $700^{\circ} \mathrm{C}$. The nominal cation composition of the film was controlled to be $\mathrm{La}: \mathrm{Sr}: \mathrm{Co}=1: 1: 2\left(\right.$ or $\left.\mathrm{La}_{0.5} \mathrm{Sr}_{0.5} \mathrm{CoO}_{3-y}\right)$.

For TEM observations, cross-sectional specimens were prepared. EDS and EELS were applied to determine the chemical composition. The average valence states of Co was determined by the ratio of white line intensities of $\mathrm{Co}_{2,3}$ ionization edges with reference to the spectra acquired from a few standard specimens with known valence states. The EDS and EELS experiments were carried out at $200 \mathrm{kV}$ using a Hitachi HF-2000 transmission electron microscope equipped with a field emission source. HRTEM, selected-area electron diff raction and dynamical electron image and diffraction pattern simulations were used to determine the structure. HRTEM experiments were performed at $300 \mathrm{kV}$ using a JEOL 3010 UHR transmission electron microscope, and the images were recorded using a charge-coupled device camera and image plates.

A low-loss valence spectrum and the corresponding core-shell ionization edge electron-energy-loss spectrum were acquired simultaneously from the same specimen region. The energy-loss spectrum was used to remove the multiple-inelastic-scattering effect in the core-loss region using the Fourier ratio technique; thus the presented data are for single scattering.

\section{§ 3. ExPERIMENTAL RESUlts}

\subsection{Chemical composition}

The chemical composition of LSCO is determined first using EDS. This analysis can be performed from a region as small as a few nanometres. To examine the 
Fig. 1

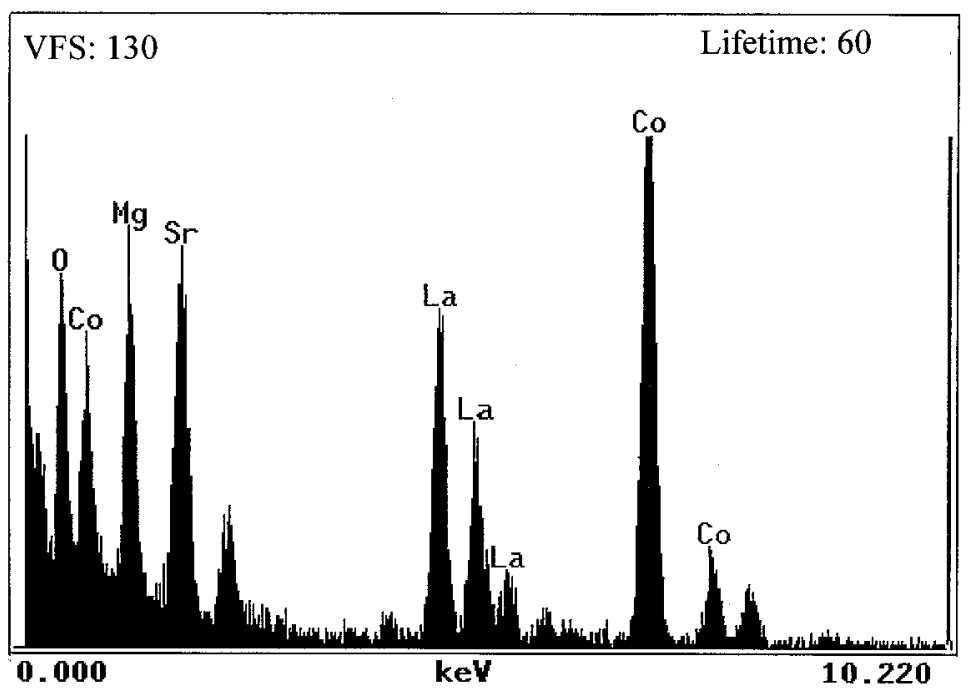

An energy-dispersive spectrum of $\mathrm{La}_{0.5} \mathrm{Sr}_{0.5} \mathrm{CoO}_{3-}$ acquired at $200 \mathrm{kV}$ showing the various elements presented in the specimen.

chemical stoichiometry across a large area, series of spectra were acquired from different regions and the results indicated that the specimen was chemically uniform. Shown in fig. 1 is a typical energy-dispersive spectrum acquired from a thin region of $\mathrm{LSCO}$ with the use of a thin-window $\mathrm{Si}$ detector. In addition to the $\mathrm{La}, \mathrm{Sr}, \mathrm{Co}$ and $\mathrm{O}$ elements from the film, the $\mathrm{Mg}$ line comes from the $\mathrm{MgO}$ substrate. After subtraction the contribution from the $\mathrm{MgO}$ substrate to the $\mathrm{O}$ line, the chemical composition of this specimen is determined to be $\mathrm{La}_{0.5} \mathrm{Sr}_{0.5} \mathrm{CoO}_{2 \cdot 2-2 \cdot 4}$. To confirm this result, EELS was applied to determine the ratio of $\mathrm{O}$ to $\mathrm{Co}$ with the use of $\mathrm{OK}$ and $\mathrm{Co}_{2,3}$ ionization edges. In the LSCO specimen, since the La L ionization edge is located about $53 \mathrm{eV}$ above the $\mathrm{Co}_{2,3}$ edge, the energy integration window was selected as $50 \mathrm{eV}$ for composition analysis as limited by the practical situation. Based on the ionization cross-sections of $\mathrm{O} \mathrm{K}$ and $\mathrm{Co}_{2,3}$ calculated using the SIGMAK and SIGMAL programs (Egerton 1996) respectively, the result is that the O-to-Co ratio is $2 \cdot 4 \pm 0 \cdot 1$, in agreement with the EDS measurement. The combination of all this information indicates that the composition of the spectrum is $\mathrm{La}_{0.5} \mathrm{Sr}_{0.5} \mathrm{CoO}_{2 \cdot 2-2 \cdot 4}$, in which the anion deficiency $y=0 \cdot 7 \pm 0 \cdot 1$. Before we proceed further, let us examine the ionic structure of this anion-deficient compound.

LSCO has a perovskite-related structure, in which the mixed valence of the cations plays a vital role in determining the properties of the material. In the literature, Co has been believed to have valences $3+$ and $4+$ in this compound. The substitution of trivalent $\mathrm{La}^{3+}$ by divalent $\mathrm{Sr}^{2+}$ is balanced by creating $\mathrm{O}$ vacancies as well as the conversion of $\mathrm{Co}^{3+}$ into $\mathrm{Co}^{4+}$ (Jonker and van Santen 1953). The ionic structure of $\mathrm{La}_{1-x} \mathrm{Sr}_{x} \mathrm{CoO}_{3-y}$ is

$$
\mathrm{La}_{1-x}^{3+} \mathrm{Sr}_{x}^{2+} \mathrm{Co}_{1-x+2 y}^{3+} \mathrm{Co}_{x-2 y}^{4+} \mathrm{O}_{3-y}^{2-} \mathrm{V}_{y}^{\mathrm{O}} \quad\left(\text { for } y \leqslant \frac{x}{2}\right),
$$


where $\mathrm{V}_{y}^{\mathrm{O}}$ stands for O vacancies (Kurata and Colliex 1993, Wang and Zhang 1996, Buseck et al. 1989). Based on this ionic structure, the average valence state of Co is expected to be

$$
\langle\mathrm{Co}\rangle_{\mathrm{VS}}=3+x-2 y \geqslant 3 .
$$

For $x=0.5$ and $y=0.7 \pm 0.1$, however, the condition $y \leqslant x / 2$ fails; thus eqn. (1) must be modified to consider lower valences of Co. If the anion deficiency $y>x / 2$, the ionic structure of LSCO could be

$$
\mathrm{La}_{1-x}^{3+} \mathrm{Sr}_{x}^{2+} \mathrm{Co}_{1+x-2 y}^{3+} \mathrm{Co}_{2 y-x}^{2+} \mathrm{O}_{3-y}^{2-} \mathrm{V}_{y}^{\mathrm{O}} \quad(\text { for } x \leqslant 2 y),
$$

where the valence conversion is assumed to be between $\mathrm{Co}^{3+}$ and $\mathrm{Co}^{2+}$, and the average valence state of $\mathrm{Co}$ is

$$
\langle\mathrm{Co}\rangle_{\mathrm{Vs}}=3+x-2 y \leqslant 3 \text {. }
$$

In the as-grown LSCO thin films, the valence state and $\mathrm{O}$ anion deficiency are usually unknown. Therefore the valence state of Co must be measured experimentally to determine which of either eqn. (1) or eqn. (3) represents the ionic configuration of the specimen. In return, the measured average valence of Co can be used to calculate the anion deficiency $y$ of LSCO, which serves as an independent study for confirming the result given by EDS.

\subsection{Valence state of Co}

In EELS, the L ionization edges of transition-metal, rare-earth and actinide compounds usually display sharp peaks at the near edge region. These threshold peaks are known as white lines. For transition metals with unoccupied $3 \mathrm{~d}$ and $4 \mathrm{~d}$ states, the white lines are observed. The unoccupied $3 \mathrm{~d}$ states form a narrow energy band, the transition of a $2 p$ state electron to the $3 \mathrm{~d}$ levels leading to the formation of white lines observed experimentally. Thus, the atomic state changes from $2 \mathrm{p}^{6} 3 \mathrm{~d}^{m}$ to $2 \mathrm{p}^{5} 3 \mathrm{~d}^{m+1}$ after the excitation of a $2 \mathrm{p}$ electron, where $m$ stands for the number of unoccupied $3 \mathrm{~d}$ states. More specifically, the $\mathrm{L}_{3}$ and $\mathrm{L}_{2}$ lines are the transition $2 \mathrm{p}^{3 / 2} \rightarrow 3 \mathrm{~d}^{3 / 1} 3 \mathrm{~d}^{5 / 2}$ and $2 \mathrm{p}^{1 / 2} \rightarrow 3 d^{3 / 2}$ respectively. EELS experiments have shown that the change in valence states of cations introduces significant changes in the ratio of the white lines, leading to the possibility of identifying the occupation number of $3 \mathrm{~d}$ or $4 \mathrm{~d}$ electrons (or cation valence states) using the measured white-line intensities in EELS (Rask et al. 1987).

EELS analysis of valence state is usually carried out with reference to the spectra acquired from standard specimens with known cation valence states (Pearson et al. 1993, Kurata and Colliex 1993). To establish the numerical relationship between the white-line intensity and the number of unoccupied d electrons, the white lines must be isolated from the background intensity and normalized. The EELS data must be processed first to remove the multiple-scattering effect via deconvolution (Egerton 1996). The empirical method for this purpose follows that of Pearson et al. (1993, 1988). In fact, the intensity ratio $I\left(\mathrm{~L}_{3}\right) / I\left(\mathrm{~L}_{2}\right)$ has little dependence on the specimen thickness and its variation is related to the valence state although the relation is not linear. If a series of electron-energy-loss spectra are acquired from several standard specimens with known valence states) an empirical plot of these data may serve as the reference for determining the valence state of the element present in a new compound. This method has been successfully demonstrated for Mn (Kurata and Colliex 1993), and it has been applied recently to determine the average valence state 
Fig. 2
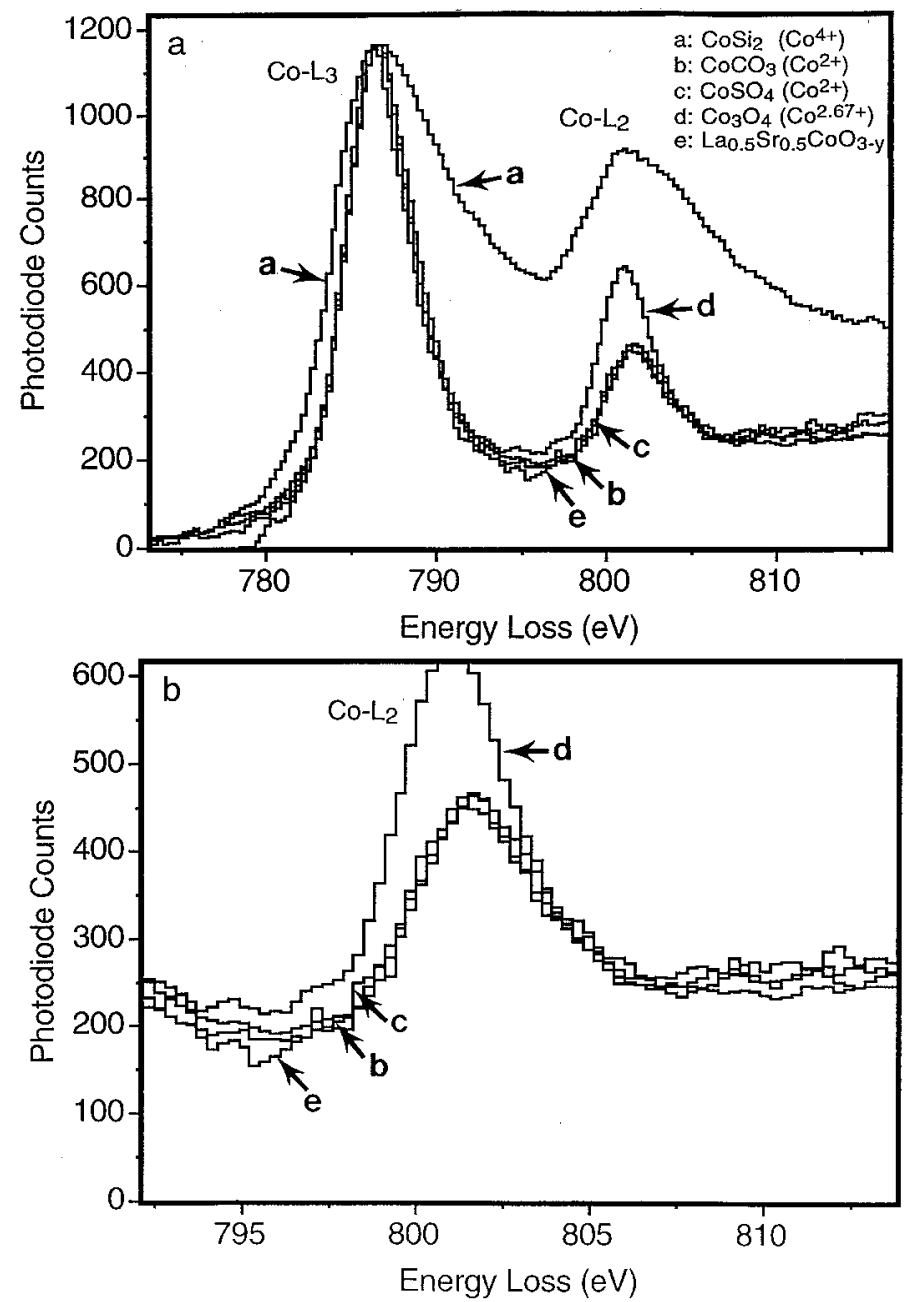

(a) A comparison of single-scattering electron-energy-loss spectra of $\mathrm{Co}_{2,3}$ ionization edges acquired from $\mathrm{CoSi}_{2}, \mathrm{CoCO}_{3}, \mathrm{CoSO}_{4}, \mathrm{Co}_{3} \mathrm{O}_{4}$ and $\mathrm{La}_{0.5} \mathrm{Sr}_{0.5} \mathrm{CoO}_{3-}$. The spectra are displayed by normalizing the heights of the $\mathrm{L}_{3}$ white lines after the subtraction of background. (b) A redisplay of the $\mathrm{Co}_{2}$ edge region for illustrating the almost identical shapes of the spectra acquired from $\mathrm{CoCO}_{3}, \mathrm{CoSO}_{4}$ and $\mathrm{La}_{0.5} \mathrm{Sr}_{0.5} \mathrm{CoO}_{3-}$, simply indicating that the valence state of $\mathrm{Co}$ in $\mathrm{La}_{0 \cdot 5} \mathrm{Sr}_{0 \cdot 5} \mathrm{CoO}_{3-y}$ is $2+$.

of $\mathrm{Mn}$ in $\mathrm{La}_{0 \cdot 67} \mathrm{Ca}_{0.33} \mathrm{MnO}_{3-y}$ (Senaris-Rodriguez and Goodenough 1995). This technique is used here to determine the average valence of Co in LSCO.

Figure 2 shows a comparison of the processed single-scattering electron-energyloss spectra of $\mathrm{Co}_{2,3}$ ionization edges acquired from $\mathrm{CoSi}_{2}$ (with $\mathrm{Co}^{4+}$ ), $\mathrm{Co}_{3} \mathrm{O}_{4}$ (with $\mathrm{Co}^{2 \cdot 67+}$ ), $\mathrm{CoCO}_{3}$ (with $\mathrm{Co}^{2+}$ ) and $\mathrm{CoSO}_{4}\left(\right.$ with $\mathrm{Co}^{2+}$ ) and $\mathrm{La}_{0.5} \mathrm{Sr}_{0.5} \mathrm{CoO}_{3-y}$. The first four compounds are chosen as the standard specimens with known Co valences, and the last is the specimen for which we wish to determine its Co valence state. It is apparent that the shape of $\mathrm{CoSi}_{2}$ (with $\mathrm{Co}^{4+}$ ) is dramatically different 
Fig. 3

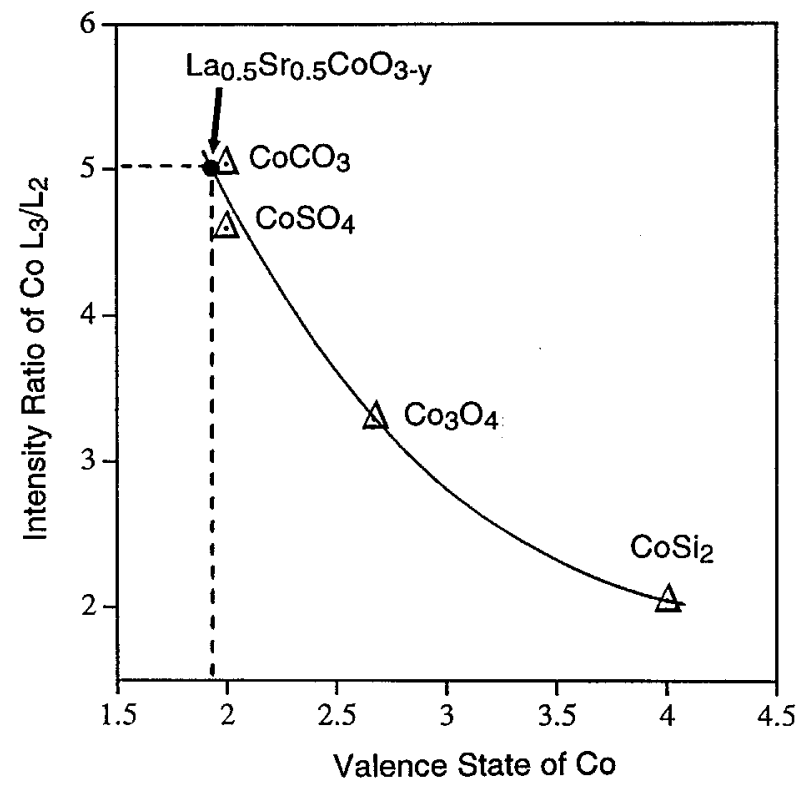

A plot of the intensity ratio $I\left(\mathrm{~L}_{3}\right) / I\left(\mathrm{~L}_{2}\right)$ calculated from the spectra shown in fig. $2(a)$ for different compounds. The Co valence state in $\mathrm{La}_{0.5} \mathrm{Sr}_{0.5} \mathrm{CoO}_{3-y}$ is obtained from the empirical fitting curve with reference to the known Co valences of the standard specimens.

from the rest not only owing to its high $\mathrm{L}_{2}$ edge but also owing to its broadened shape, simply because of its highest Co valence state. The other four specimens have the same linewidth and intensity except that $\mathrm{Co}_{3} \mathrm{O}_{4}$ (with $\mathrm{Co}^{2 \cdot 67+}$ ) has a higher $\mathrm{L}_{2}$ edge; this is because of its higher Co valence. The two standard specimens showing the same shape of $\mathrm{Co}_{2,3}$ edges have $\mathrm{Co}^{2+}$. These spectra clearly establish the experimental basis of using the white line intensities for determination the Co valence in a new compound. Following the procedures introduced by Pearson et al. (1993), the background underneath the $\mathrm{L}_{2}$ and $\mathrm{L}_{3}$ white lines is subtracted, and the intensity ratios $I\left(\mathrm{~L}_{3}\right) / I\left(\mathrm{~L}_{2}\right)$ for all the four standard specimens are plotted in fig. 3, which clearly shows that $I\left(\mathrm{~L}_{3}\right) / I\left(\mathrm{~L}_{2}\right)$ is very sensitive to the valence state of Co. From the empirical fitting curve, the valence of $\mathrm{Co}$ in $\mathrm{La}_{0.5} \mathrm{Sr}_{0.5} \mathrm{CoO}_{3-y}$ can be determined from its $I\left(\mathrm{~L}_{3}\right) / I\left(\mathrm{~L}_{2}\right)$ value $(5 \cdot 05)$. The corresponding horizontal axis is approximately 1.93 , which means that the valence of $\mathrm{Co}$ in $\mathrm{La}_{0.5} \mathrm{Sr}_{0.5} \mathrm{CoO}_{3-y}$ is $2+$ on consideration of the experimental error. Therefore the ionic structure of LSCO is described by eqn. (3) with $y=(x+1) / 2=0.75$ which is

$$
\mathrm{La}_{0 \cdot 5}^{3+} \mathrm{Sr}_{0.5}^{2+} \mathrm{Co}^{2+} \mathrm{O}_{2 \cdot 25}^{2-} \mathrm{V}_{0 \cdot 75}^{\mathrm{O}}
$$

Therefore the compound is chemically non-stoichiometric with the composition of $\mathrm{La}_{0.5} \mathrm{Sr}_{0.5} \mathrm{CoO}_{2 \cdot 25}$, in agreement with the results obtained from EDS and EELS microanalysis (see section 3.1). 


\subsection{High-resolution transmission electron microscopy lattice image of

$$
\mathrm{La}_{1-x} \mathrm{Sr}_{x} \mathrm{CoO}_{3-y}
$$

With the known chemistry of this compound, we now determine the crystal structure with an emphasis on the anion atoms in the compound. Traditionally, $\mathrm{X}$-ray and neutron diffraction are probably the most precise techniques for determining the structure of large single crystals. For MOCVD-grown LSCO thin films, however, the crystal is small and the influence from the substrate is enormous because of high-density interface mismatch dislocations. HRTEM is likely to be a unique technique for this case.

Figure 4 shows a low-magnification TEM image of the specimen. It is apparent that the film exhibits domain structure, and the $c$-axis direction changes from domain to domain. The domain size is approximately $50-300 \mathrm{~nm}$. The three-dimensional (3D) structure of the film is composed of domains with [001] [010] and [100] directional anisotropy, as indicated by the arrows. The $c$-axes of some domains are in the image plane ( $a-b$ plane) while the others with the $c$ axis pointing into the paper. The presence of domains makes the structure analysis using either X-ray or neutron diffraction unfeasible because of the average effect over the domains oriented along $x, y$ and $z$ directions. This is probably the reason why this phase was not found via X-ray diffraction (Galasso 1990). An inset shown in fig. 4 is a selected-area electron diffraction pattern recorded from the film. In addition to the allowed reflections of the basic $\mathrm{ABO}_{3}$-type perovskite structure, a remarkable phenomenon is the presence of (002) reflections of LSCO. It must be pointed out here that the indices are given in reference to the structure model to be illustrated later in fig. $9(a)$.

Fig. 4

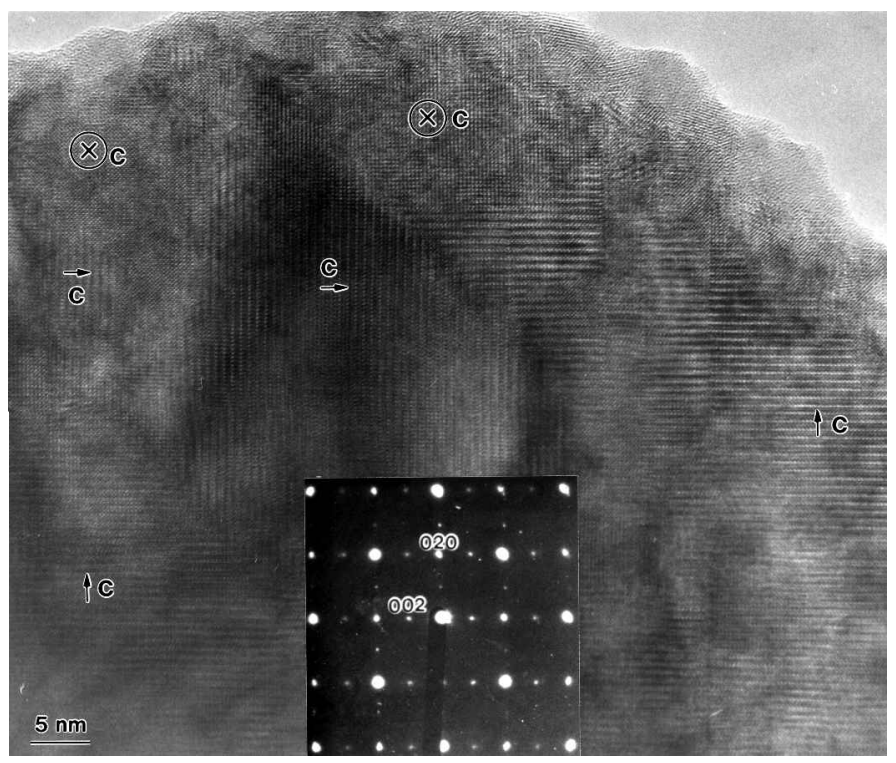

A low-magnification cross-section TEM image of $\mathrm{La}_{0.5} \mathrm{Sr}_{0.5} \mathrm{CoO}_{2.25}$ showing anisotropic domain structures, where the arrows indicate the $c$-axis directions in the local regions. The corresponding electron diffraction pattern from the film is shown in the inset. 
Fig. 5

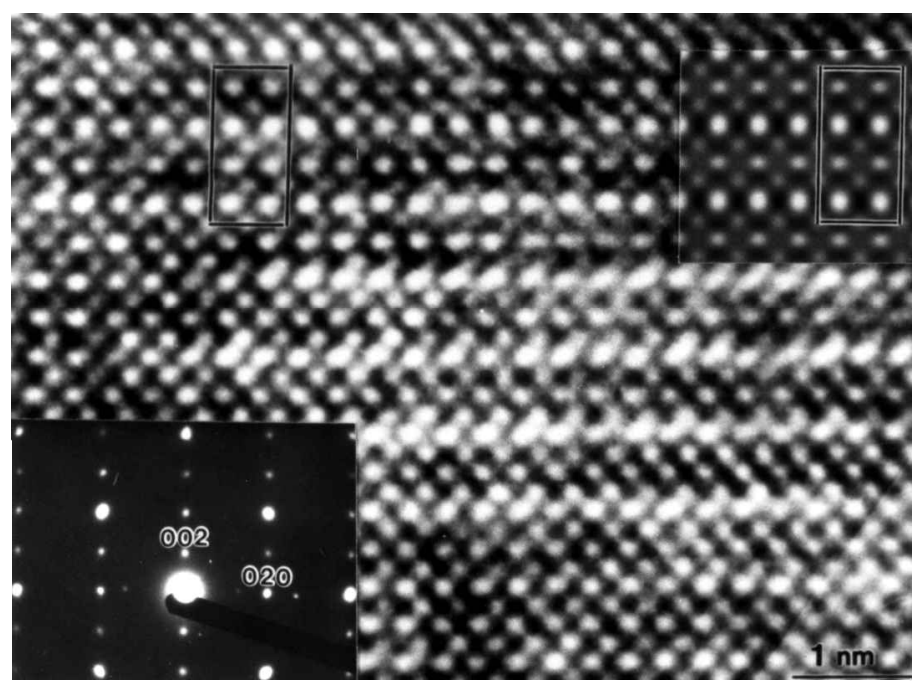

A high-magnification [100]TEM image of $\mathrm{La}_{0.5} \mathrm{Sr}_{0.5} \mathrm{CoO}_{2 \cdot 25}$, where the white spots correspond to the projected atom columns with La the strongest contrast, $\mathrm{Sr}$ strong, Co weak and $\mathrm{O}$ invisible. The inset in the lower left corner is an electron diffraction pattern from the region. The inset in the upper right corner is a theoretically simulated image based on the structure model to be shown in fig $9(a)$ for the following parameters: electron beam energy, $300 \mathrm{eV}$; specimen thickness, $3.88 \mathrm{~nm}$; beam convergence, $0.3 \mathrm{mrad}$; mechanical vibration of the microscope, $0.03 \mathrm{~nm}$; objective lens defocus, $15 \mathrm{~nm}$; focus spread, $10 \mathrm{~nm}$; objective lens spherical aberration, $0.6 \mathrm{~mm}$; objective aperture radius, $1 \cdot 0 \AA^{-1}$. The rectangular boxes indicate the [100] projection of the unit cell.

Figure 5 shows a high-magnification TEM image of a single domain as viewed along [100], exhibiting a $c$-axis directional anisotropic structure. A corresponding electron diffraction pattern from a single domain is also shown, in which the (002) reflection is present but the (010) reflection is extinct. These images are most sensitive to the cation positions in the specimen but not $\mathrm{O}$ owing to its weak scattering power. The image is also insensitive to the valence state of Co. The structure symmetry is either tetragonal or orthorhombic (with $a \approx b$ ), and the cation distribution can be directly derived from the image if the imaging conditions are known.

To get a 3D view of the structure, images along [001] are also recorded, as shown in fig. 6 , in which no anisotropic structure is observed. The image has a periodicity of $a / 2$ along the $a$-axis and $b / 2$ along the $b$-axis (with $a \approx b$ ) and the image contrast fits well to the $\mathrm{ABO}_{3}$ perovskite. An electron diffraction pattern in this orientation is also shown in the inset, exhibiting the symmetry of the [001] cubic-like diff raction pattern and the extinction of the (100) and (010) reflections. This image is distinctly different from that recorded along [100], suggesting an anisotropic structure of the crystal. Based on these HRTEM images, the cation lattice can be determined. With reference to the anion deficiency, we may start from a perovskite-related structure without an anion deficiency to work out the real structure model of this phase. This is the strategy for the following analysis. 
Fig. 6
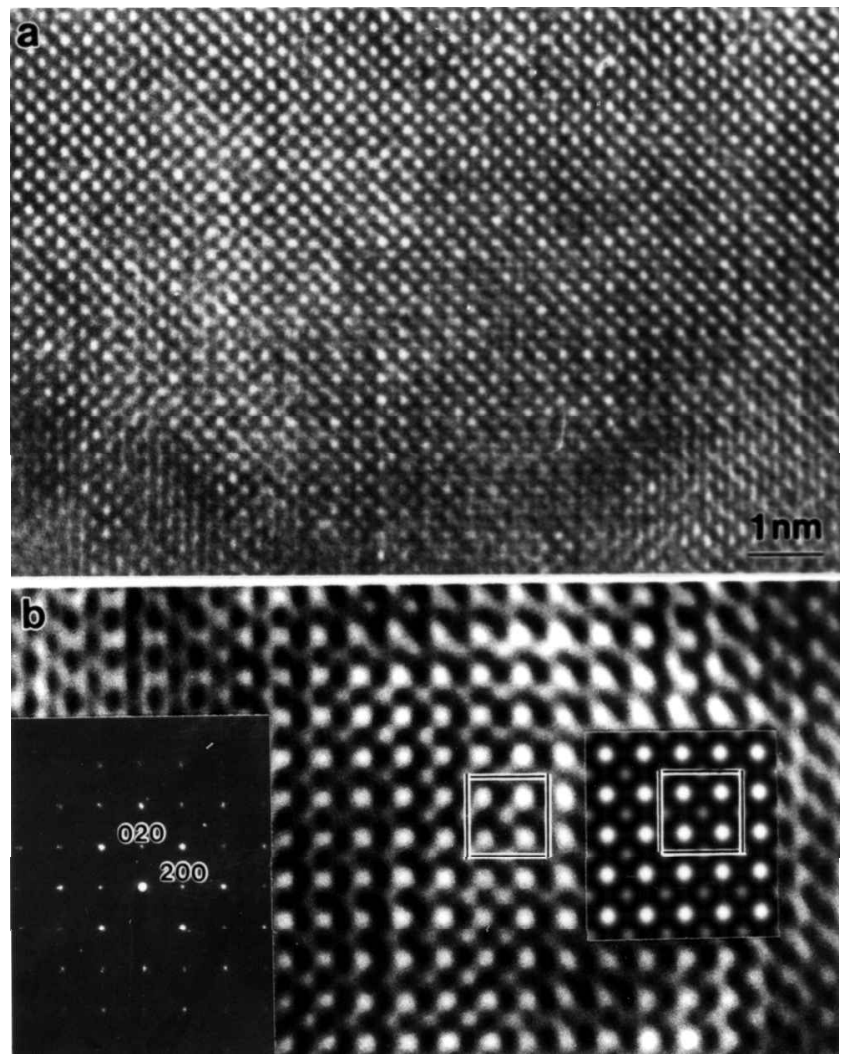

(a) A high-magnification [001] TEM image of $\mathrm{La}_{0.5} \mathrm{Sr}_{0.5} \mathrm{CoO}_{2.25}$ showing a square-shaped projected unit cell along the $c$ axis, where the white spots correspond to the projected atom columns. (b) An enlarged [001] image and the corresponding electron diffraction pattern. The inset is a theoretically simulated image based on the structure model to be shown in fig. $9(a)$ for the following parameters: electron beam energy, $300 \mathrm{keV}$; specimen thickness, $3.66 \mathrm{~nm}$; beam convergence, $0.3 \mathrm{mrad}$; mechanical vibration of the microscope, $0.02 \mathrm{~nm}$; objective lens defocus, $15 \mathrm{~nm}$; focus spread, $10 \mathrm{~nm}$; objective lens spherical aberration $0.6 \mathrm{~mm}$; objective aperture radius, $1 \cdot 0 \mathrm{~A}^{-1}$. The square boxes indicate the $[100]$ projection of the unit cell.

All the experimental data indicate that the crystal is either tetragonal or orthorhombic (with $a \approx b$ ). In order to verify this result, X-ray diffraction was performed on an as-grown film and the result is shown in fig. 7. The (002) reflection, although weak, does show up in the diffraction spectrum. It is noticed that the reflection angle of (200) is slightly less than that of (004), indicating that the LSCO has a structure with $c / a=1.89$ and $a \approx b$. The presence of the (002) reflection in the $\mathrm{X}$-ray diffraction spectrum indicates that the observed LSCO structure is the asgrown structure of the specimen, excluding the artefacts arising from TEM specimen preparation. 
Fig. 7

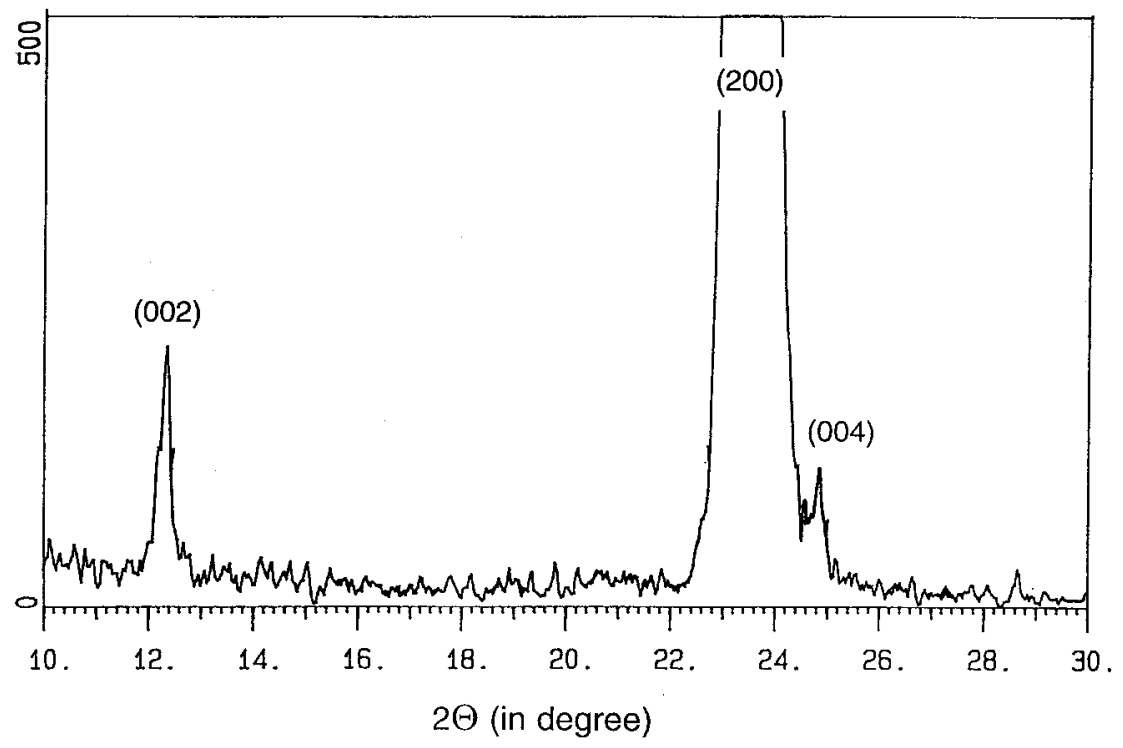

X-ray diffraction of $\mathrm{La}_{0.25} \mathrm{Sr}_{0.5} \mathrm{CoO}_{2.25} / \mathrm{MgO}$ showing the presence of (002) and (004) reflections. It is important to note that the (200) reflection angle is slightly less than that of (004), indicating that $c<2 a$.

\subsection{Structure model of $\mathrm{La}_{0.5} \mathrm{Sr}_{0.5} \mathrm{CoO}_{2 \cdot 25}$}

As a summary of the analysis above, the crystal structure of $\mathrm{La}_{0.5} \mathrm{Sr}_{0.5} \mathrm{CoO}_{2 \cdot 25}$ satisfies the following conditions:

(1) The chemical composition is $\mathrm{La}_{0.5} \mathrm{Sr}_{0.5} \mathrm{CoO}_{2.25}$ and the genetic structure formula is $\mathrm{La}_{8} \mathrm{Sr}_{8} \mathrm{Co}_{16} \mathrm{O}_{36}$.

(2) From the X-ray diffraction data, the unit cell is either tetragonal or orthorhombic with $c / a \approx 1.89$, and lattice constants $a \approx b=0.77 \mathrm{~nm}$ and $c=1.455 \mathrm{~nm}$.

(3) The valence state of $\mathrm{Co}$ is $2+$.

(4) The $\{100\}$ - and $\{110\}$-type reflections are extinct in the [001] electron diffraction pattern, indicating that the [001] projection of the unit cell has an almost square shape with a periodicity of $a / 2$ along [100] and $b / 2$ along [010]

(5) The (100)- and (001)-type reflections are extinct in the [100] electron diffraction pattern, indicating that the [100] projection of the unit cell has a rectangle shape with a periodicity of $b / 2$ along the $b$-axis and $c / 2$ along the $c$-axis.

(6) The calculated image according to the model must fit the contrast observed in both [100] and [001] HRTEM images. The [100] (or [010]) projection of the structure model need to show a layered structure of $\mathrm{La}-\mathrm{Co}-\mathrm{Sr}-\mathrm{Co}-\mathrm{La}-$ $\mathrm{Co}-\mathrm{Sr}-\mathrm{Co}-$ parallel to the (001) plan in reference to the image contrast shown in fig. 5.

(7) The anion coordination of the cations must be able to screen the Coulomb interaction from the neighbouring cations to preserve the stability of the 
Fig. 8

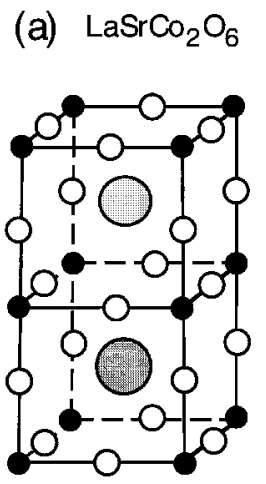

(b) $\mathrm{LaSrCO}_{2} \mathrm{O}_{4.5}$ modules
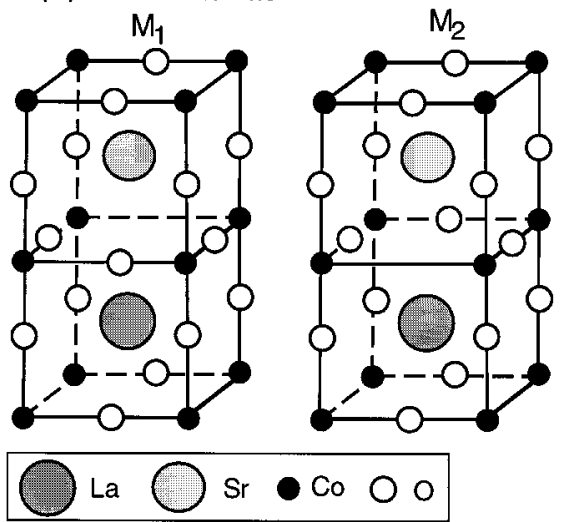

(c) $\mathrm{La}_{8} \mathrm{Sr}_{8} \mathrm{Co}_{16} \mathrm{O}_{36}$

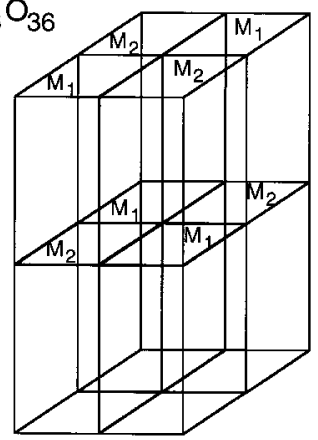

(a) A structure model of $\mathrm{LaSrCo}_{2} \mathrm{O}_{6}$ based on the previous studies of $\mathrm{La}_{0.5} \mathrm{Sr}_{0.5} \mathrm{CoO}_{3}$ grown on $\mathrm{LaAlO}_{3}(001)$ (Wang and Zhang 1996). (b) Two anion-deficient modules of $\mathrm{LaSrCoO}_{2} \mathrm{O}_{4.5}$. (c) The corresponding stacking to a form a complete unit cell of $\mathrm{La}_{8} \mathrm{Sr}_{8} \mathrm{Co}_{16} \mathrm{O}_{36}$ ( or $\mathrm{La}_{0.5} \mathrm{Sr}_{0.5} \mathrm{CoO}_{2 \cdot 25}$ ).

structure. The Co coordination number, for example, can be $3,4,5$ or 6 . If the coordination number is 3 , the three anions must be in the same plane with the Co cation. The four-coordinated Co cation is possible if either it is surrounded by four cations in the same plane or the four form a tetrahedron. This is an extremely important rule with which the structure must comply.

To begin the construction of the model, we start from a fundamental stoichiometric module of $\mathrm{LaSrCo}_{2} \mathrm{O}_{6}$ ( or $\mathrm{La}_{0.5} \mathrm{Sr}_{0.5} \mathrm{CoO}_{3}$ ) without anion deficiency, as shown in fig. 8(a), which is a combination of two perovskite unit cells of $\mathrm{LaCoO}_{3}$ and $\mathrm{SrCoO}_{3}$, and this model was proposed previously in our studies of LSCO grown on $\mathrm{LaAlO}_{3}$ (Wang and Zhang 1996). The new structure unit is composed of eight modules of this type but with ordered anion vacancies in each. The chemical composition of each module is required to be $\mathrm{LaSrCo}_{2} \mathrm{O}_{4 \cdot 5}$, where the non-integer $\mathrm{O}$ anions means that an $\mathrm{O}$ vacancy is shared by at least two modules; thus at least two different types of module must be created. The positions of $\mathrm{O}$ vacancies in the 
Fig. 9

(a)

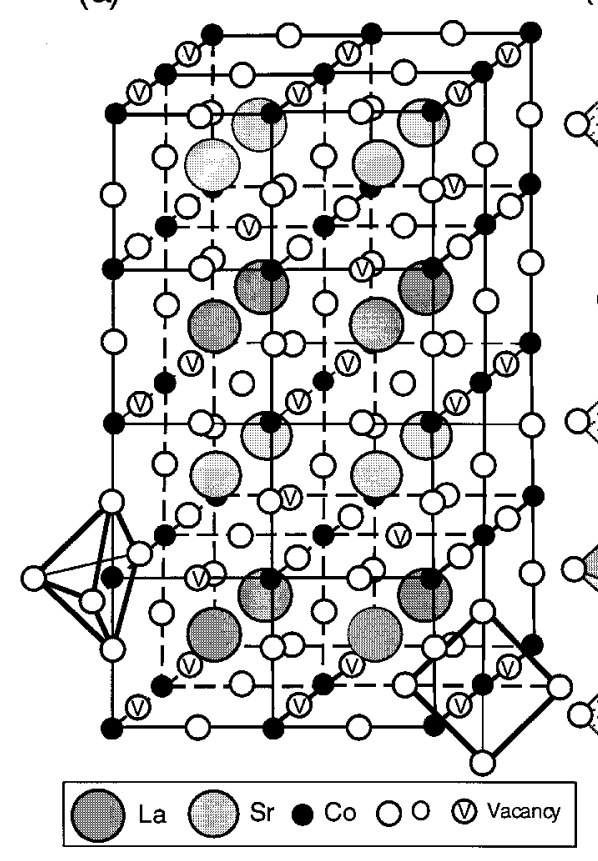

(c)
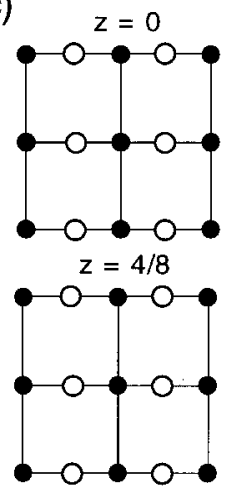
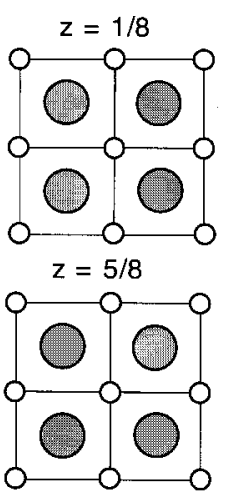

(b)

b)

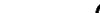

a

R

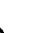

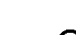


$\mathrm{LaCoO}_{3-\delta}$ basic perovskite unit cells. Divalent $\mathrm{Co}$ is likely to be coordinated by one $\mathrm{O}$ atom on average in the top and bottom layers.

These modules are the building blocks for constructing the full unit cell of LSCO, as shown for $\mathrm{La}_{8} \mathrm{Sr}_{8} \mathrm{Co}_{16} \mathrm{O}_{36}$ in fig. 8(c); here the subscripts represent the numbers of corresponding atoms in the cell. This unit cell is orthorhombic $(a \approx b)$. The cations are distributed in layered structure along the $c$-axis. The 3D atom distribution in the full unit cell is given in fig. 9(a). The coordination numbers of $\mathrm{La}$ and $\mathrm{Sr}$ are 9, and those of Co are 5 and 4 (fig. $9(a)$ ); thus the structure is chemically stable. The five $\mathrm{O}$ coordinated $\mathrm{Co}$ is a half-octahedron (e.g. a square-based pyramid) and the four $\mathrm{O}$ coordinated $\mathrm{Co}$ is a square sheet. These units are the fundamental blocks for building the structure (fig. $9(b)$ ). The atom distributions in layers perpendicular to the $c$-axis are given in fig. $9(c)$. We have tried a variety of module configurations and this type of arrangement is the only one that can fit all our requirements listed above.

If the structure is projected along $[100]$ the projected structure has a periodicity of $b / 2$ along [010] and $c / 2$ along [001] thus both the (010) and the (001) reflections are forbidden. The projection of this structure along [001] has a periodicity of $a / 2$ along [100] and $b / 2$ along [010] and the $a$ and $b$ axis are not identical; because of a difference in the projected $\mathrm{O}$ atoms, even the cation lattice is symmetric; thus the (100) and (010) reflections are forbidden. In practice, the difference between the $a$ and $b$ axes is so small that it cannot be detected using electron diffraction for a case in which the domain structure is present in particular.

In order to verify the atomic model, [100] and [001] HRTEM images are simulated and they are shown in the insets of fig. 5 and $6(b)$ respectively. The calculations were based on the dynamical electron diffraction theory with considerations of the contrast transfer of the objective lens as well as the practical operation conditions of the electron microscopy (Buseck et al. 1989). The image contrast is approximately scaled according to the atomic number, as expected from the projected-chargedensity approximation for thin specimens. The La atom shows the strongest intensity, the $\mathrm{Sr}$ atom weaker and the $\mathrm{Co}$ atom the weakest, but the $\mathrm{O}$ atoms are unresolved. The projections of the unit cell along [100] and [001] are indicated in figs. 5 and 6 respectively on the observed and the simulated images. Excellent agreement is obtained.

Figures $10(a)$ and $(c)$ are the dynamical calculated electron diffraction patterns for [100] and [001] and figs. $10(b)$ and $(d)$ are the experimentally observed diffraction patterns respectively. The (001) and (100) reflections are extinct, in agreement with the experimental observations. Electron diff raction is very sensitive to the symmetry of the crystal. A site change of a single $\mathrm{O}$ atom in the cell can break the symmetry, giving the (100)-type reflection in the calculated pattern. This is the method that we used to exclude the models that fit the HRTEM images but not the diffraction patterns because the images are dominated by the scattering of cations while the diff raction pattern is most sensitive to the symmetry. Our calculation shows that the model proposed in fig. $9(a)$ for all the available experimental data is the unique solution.

\section{$\S 4$. Discussion}

\subsection{The measurement of the Co ionization state}

It has been demonstrated by several workers that the $3 \mathrm{~d}$ and $4 \mathrm{~d}$ electron occupancies can be more precisely determined with the use of the normalized white-line 
Fig. 10

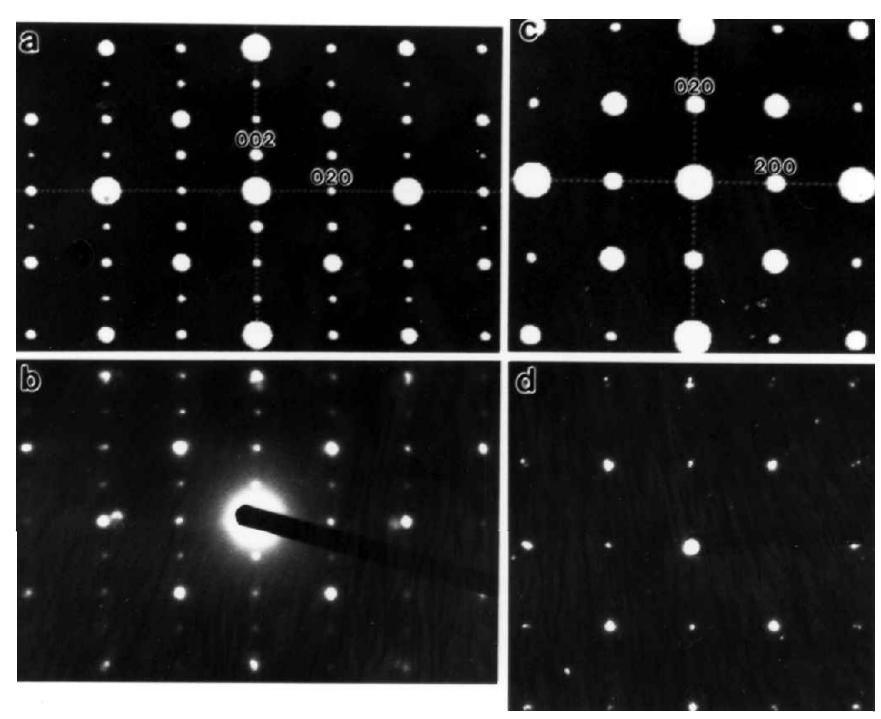

(a), (c) Theoretically calculated electron diffraction patterns based on the model in fig. $9(a)$. $(b),(d)$ The corresponding observed electron diffraction patterns for [100] and [001] orientations respectively, showing good agreement. The double split of the pattern shown in $(d)$ is due to the lattice mismatch between LSCO and MgO substrate.

intensity and the continuous spectrum at $50-100 \mathrm{eV}$ above the edge threshold (Pearson et al. 1983, 1993, Kurata and Colliex 1993). In practice, however, the intensity in the region 50-100 eV above the edge threshold may be affected by the deconvolution and spectrum background subtraction procedures particularly when the noise level and gain variation are significant. Moreover, for $\mathrm{La}_{0.5} \mathrm{Sr}_{0.5} \mathrm{CoO}_{2.25}$, the $\mathrm{La} \mathrm{L}_{2,3}$ edge is located at $832 \mathrm{eV}$, which is only $53 \mathrm{eV}$ above the $\mathrm{Co}_{2,3}$ edge; thus the strong overlap of the two ionization edges prohibits accurate calculate of the continuous component above the edge threshold. In contrast, the intensity ratio $I\left(\mathrm{~L}_{3}\right) / I\left(\mathrm{~L}_{2}\right)$ has little dependence on the specimen thickness and its variation can also be related to the valence state although the relation is not linear. If a series of electron-energy-loss spectra are acquired from several standard specimens with known valence states, an empirical plot of these data should serve as the reference for determining the valence state of the element present in a new compound. This has been demonstrated in our study.

It has also been pointed out in the literature that the $I\left(\mathrm{~L}_{3}\right) / I\left(\mathrm{~L}_{2}\right)$ ratio is approximately the ratio of the electrons in the $j=\frac{5}{2}$ state to those in the $j=\frac{3}{2}$ state; thus the white-line intensity may be sensitive to the spin distribution (Lloyd et al. 1995, Yuan et al. 1994). We now consider the difference made by the electron distribution in different spin states. From the electron-energy-loss spectra of the four standard specimens, $\mathrm{CoCO}_{3}, \mathrm{CoSO}_{4}, \mathrm{CoSi}_{2}$ and $\mathrm{Co}_{3} \mathrm{O}_{4}$ (with $\mathrm{Co}^{2 \cdot 67+}$ ) (see fig. 2), the former two with $\mathrm{Co}^{2+}$ show almost identical $\mathrm{Co} \mathrm{L}_{2,3}$ shapes, while the last two with $\mathrm{Co}^{4+}$ and $\mathrm{Co}^{2 \cdot 67}$ show distinct difference in the $\mathrm{Co} \mathrm{L}_{2}$ shapes. The small difference between the $I\left(\mathrm{~L}_{3}\right) / I\left(\mathrm{~L}_{2}\right)$ ratios of $\mathrm{CoCO}_{3}$ and $\mathrm{CoSO}_{4}$ in fig. 3 might be due to the spin effect, but this small fluctuation cannot significantly affect the measurement. 
This indicates that, at least in our case, the electron distribution in spin states, if any, plays a negligible role.

\subsection{The oxygen stoichiometry}

In the anion-deficient compound $\mathrm{La}_{0.5} \mathrm{Sr}_{0.5} \mathrm{CoO}_{2 \cdot 25}$ (or $\mathrm{La}_{8} \mathrm{Sr}_{8} \mathrm{Co}_{16} \mathrm{O}_{36}$ ), if the $\mathrm{O}$ content is less than $2 \cdot 25$, the structure cannot exist because the ionic charge is not balanced since the lowest valence state of Co is $2+$. Thus the compound presented here may be the least stable compound in the $\mathrm{La}-\mathrm{Sr}-\mathrm{Co}-\mathrm{O}$ system and it may not grow into a large single crystal. This might be the reason why small domains are formed in the film. Amazingly, a specimen of this compound has found to be stable in air for 2 years after growth and the same crystal structure is preserved. The $\mathrm{MgO}$ substrate may help to maintain the stability of the specimen. This is a key difference between thin-film materials and large bulk crystals.

To see the sensitivity of the Co valence state to anion deficiency, we carry out the following exercise. If the $\mathrm{O}$ content in each module shown in fig. $9(a)$ was increased by $0 \cdot 5$, so that the composition would be $\mathrm{LaSrCo}_{2} \mathrm{O}_{5}$ or $\mathrm{La}_{0.5} \mathrm{Sr}_{0.5} \mathrm{CoO}_{2 \cdot 25}$, the ionic structure of this phase would be

$$
\mathrm{La}_{0.5}^{3+} \mathrm{Sr}_{0.5}^{2+} \mathrm{Co}_{0 \cdot 5}^{3+} \mathrm{Co}_{0 \cdot 5}^{2+} \mathrm{O}_{2 \cdot 5}^{2-}
$$

In comparison with the ionization state given by eqn. (5) for $x=0.5$ and $y=0 \cdot 75$, namely

$$
\mathrm{La}_{0.5}^{3+} \mathrm{Sr}_{0.5}^{2+} \mathrm{Co}^{2+} \mathrm{O}_{2 \cdot 25}^{2-}
$$

the average valence of Co changes from $2+$ for $y=0.75$ to $2 \cdot 5+$ for $y=0 \cdot 5$. This drastic change in average valence can be sensitively measured using EELS based on the data presented in fig. 3. Therefore the valence state provided by EELS is very sensitive to a small change in anion deficiency, provided that the structure is ordered.

The cation lattice in the proposed model agrees exactly with our previous study on the tetragonal cation lattice in $\mathrm{La}_{0.5} \mathrm{Sr}_{0.5} \mathrm{CoO}_{3}$ grown on $\mathrm{LaAlO}_{3}(001)$ (Wang and Zhang 1996), which was derived solely on the basis of HRTEM data. The ordered structure has a tetragonal cell with a $\mathrm{La}-\mathrm{Co}-\mathrm{Sr}-\mathrm{Co}-(001)$ layered atom distribution perpendicular to the $c$ axis. This structure is intrinsic for the LSCO system and resulted directly from the lattice substitution between La and Sr. The key difference between the current study and the previous study is that the anion positions are determined. In fact, this is probably the first example of using EELS data together with HRTEM to determine the ordered point defects in complex crystal structures. This study is expected to lead to a new method for studying anion-deficient oxide functional materials.

\subsection{Anion deficiency, structure evolution and colossal magnetoresistance effect}

As pointed out at the beginning, ( $\mathrm{La}, \mathrm{A}) \mathrm{MnO}_{3}$ type of compounds have been found to exhibit the CMR effect (Zhang et al. 1992, Chahara et al. 1993, Jin et al. 1994, Ju et al. 1994, Von Helmolt et al. 1994, Li et al. 1995) while the CMR effect of ( $\mathrm{La}, \mathrm{A}) \mathrm{CoO}_{3}$ is dramatically small (Briceno et al. 1995). There are several factors which may account for this difference. For $\mathrm{La}_{0.67} \mathrm{Ca}_{0.33} \mathrm{MnO}_{3-\text { y }}$ (LCMO), the unit cell is still the $\mathrm{ABO}_{3}$ type without a superstructure; thus the interlayer isolation is preserved (Wang et al. 1996). The ratio of $\mathrm{Mn}^{4+}$ to $\mathrm{MN}^{3+}$ is measured to be more than $0 \cdot 25$ but less than $0 \cdot 5$, and the $\mathrm{O}$ vacancy content $y$ is no more than 0.065 (equivalent to $2 \cdot 2$ at. $\%$ O content) (Wang et al. 1997). Thus the double-exchange (or 
electron double-hopping) process (Zener 1951) is possible in LCMO, as described in following. The electron hopping away from an $\mathrm{Mn}^{3+}$ atom remembers the spin state that it has on the ion as determined by Hund's rule, and the electron hopping onto the $\mathrm{Mn}^{4+}$ must have the same spin state. This is only possible, without violating Hund's rule on the $\mathrm{Mn}^{4+}$, if the net ion spins of the neighbouring $\mathrm{Mn}^{3+}$ and $\mathrm{Mn}^{4+}$ are in the same spin direction. The hopping is impossible if the spin directions of the two states are antiparallel. In fact, the likelihood of electron hopping between two magnetic ions turns out to depend on the spinor transformation (Anderson and Hasegawa 1995, deGennes 1960) which is characterized by a transfer probability

$$
t_{i j}=b_{i j} \cos \left(\frac{\theta_{i j}}{2}\right),
$$

where $b_{i j}$ is a constant depending on the isolation between the ions and $\theta_{i j}$ is the angle between the direction of the spin ions. Thus the electrical resistance of the material is a function of its intrinsic magnetic order. The angle between the spins of two ions will be changed under an external magnetic field. Thus the electron transfer probability across the ions (or equivalently the conductivity) is affected according to eqn. (8).

Structural evolution of LSCO introduces changes not only in the intrinsic isolation but also in the spin coupling between Co ions. Missing O anions in LSCO may reduce the interlayer isolation between Co. This could strongly reduce the CMR effect of this material since the CMR effect is assumed to be due to a doubleexchange process of electron hopping. Moreover, for LSCO with a single-valence $\mathrm{Co}^{2+}$, no double exchange would occur; thus the material may not exhibit the CMR effect, in agreement with the observation of Briceno et al. (1995) for $x=0.5$ alternatively the CMR might be caused by a different mechanism.

On the other hand, $\mathrm{La}_{0.5} \mathrm{Sr}_{0.5} \mathrm{CoO}_{3-y}$ show a domain structure and superlattice, while $\mathrm{La}_{0.67} \mathrm{Ca}_{0.33} \mathrm{MnO}_{3}$ has the $\mathrm{ABO}_{3}$ structure (Wang et al. 1996). The CMR effect of LSCO, if any, may be critically affected by the domain structure. Although each domain may exhibit a high CMR ratio, the overall CMR ratio of the entire film may not be high because the small anisotropic domains are distributed with equal probability along the $x, y$ and $z$ directions; the spatial average may reduce the CMR ratio (Wang and Zhang 1996).

\section{§5. CONCLUSION}

In this paper, the crystal structure of an anion-deficient orthorhombic perovskite-type phase $\mathrm{La}_{0.5} \mathrm{Sr}_{0.5} \mathrm{CoO}_{2.25}$ (or $\mathrm{La}_{8} \mathrm{Sr}_{8} \mathrm{Co}_{16} \mathrm{O}_{36}$ ) has been determined. The specimen composition was first measured using EDS and EELS to quantify the anion deficiency. The Co valence state is measured to be $2+$ using the white-line intensities in EELS with reference to the spectra acquired from several standard specimens with known valence states. The anion deficiency calculated on the basis of this result agrees well with the EDS result. Then the cation lattice was determined using HRTEM for both [100] and [001] orientations. Based on this information and the structure symmetry provided by electron diffraction data, an anion-deficient structural model was proposed. The model is made out of two types of fundamental module and it is composed of a total of eight modules. The model still preserves the characteristics of perovskite framework and it is a superstructure induced by $\mathrm{O}$ vacancies. Each module is a $c$-axis stacking of the anion-deficient $\mathrm{SrCoO}_{3-z}$ and $\mathrm{LaCoO}_{3-\delta}$ basic perovskite cells. The observed new superlattice may account for the small CMR effect of LSCO. 
This study clearly illustrates the correlation of anion deficiency with the valence state of $\mathrm{Co}$, and it also proves that the $\mathrm{O}$ atom positions can be determined using the combination of EDS, EELS, HRTEM and electron diffraction. This is likely to be an effective method for probing the microstructure of perovskite-like functional materials, in which $\mathrm{O}$ vacancies play a key role in determining the structural evolution and its functionality.

\section{ACKNOWLEDGEMENTS}

Thanks are due to Dr Jiming Zhang for kindly providing the specimens used in this study and Dr Z. C. Kang for a stimulating discussion. This work was supported in part by National Science Foundation grant No. DMR-9632823.

\section{REFER ENCES}

Anderson, P. W., and Hasegawa, H., 1955, Phys. Rev., 100, 675.

Bric eno, G., Xiang, X.-D., Change, H., Sun, X., and Schultz, P. G., 1995, Science, 270, 273.

Buseck, P., Cowley, J., and Eyring, L. (editor), 1989, High-Resolution Transmission Electron Microscopy and Associated Techniques (Oxford University Press).

Chahara, K., Ohno, T., Kasai, M., and Kozono, Y., 1993, Appl. Phys. Lett., 63, 1990.

DeGennes, P. G., 1960, Phys. Rev., 118, 141.

Egerton, R. F., 1996, Electron Energy-Loss Spectroscopy in the Electron Microscopy, Second edition (New York: Plenum).

Galasso, F. S., 1990, Perovskites and High $T_{\mathrm{c}}$ Superconductors (New York: Gordon and Breach).

Jin, J., Tiefel, T. H., McCormack, M., Fatnacht, R. A., Ramech, R., and Chen, L. H., 1994, Science, 264, 413.

Jonker, J. H., and van Santen, G. H., 1953, Physica, 19, 120.

Ju, H. L., Kwon, C., Greenne, R. L., and Venkatessan, T., 1994, Appl. Phys. Lett., $65,2108$.

Kurata, H., and Colliex, C., 1993, Phys. Rev. B, 48, 2102.

Li, Y. Q., Zhang, J., Pombrik, S., DiMascio, S., Stevens, W., Yan, Y. F., and Ong, N. P., 1995, J. Mater. Res., 10, 2166.

Lloyd, S. J., Botton, G. A., and Stobis, W. M., 1995, J. Microsc., 180, 288.

Pearson, D. H., Ahn, C. C., and Fultz, B., 1993, Phys. Rev. B, 47, 8471.

Pearson, D. H., Fultz, B., and Ahn, C. C., 1988, Appl. Phys. Lett., 53, 1405.

Prinz, G. A., 1995, Phys. Today, April, 58.

Rask, J. H., Mine, B. A., and Buseck, P. R., 1987, Ultramicroscopy, 32, 319.

Senarís-Rodriguez, M. A., and Goodenough, J. B., 1995, J. solid-st. Chem., 118, 323.

Von Helmolt, R., Wecker, J., Holz a pfel, B., Schultz, L., and Samwer, K., 1994, Phys. Rev. Lett., 71, 2331.

Wang, Z. L., and Kang, Z. C., 1997, Functional and Smart Materials-Structural Evolution and Structure Analysis (New York, Plenum).

Wang, Z. L., Y In, J. S., Jiang, Y. D., and Zhang, J., 1997, Appl. Phys. Lett., 70, 3362.

Wang, Z. L., and Zhang, J., 1996, Phys. Rev. B, 54, 1153.

Wang, Z. L., Zhang, J., and Berta, Y., 1996, Epitaxial Oxide Thin Films II, Materials Reseach Society Symposium Proceedings, Vol. 401, edited by J. S. Speck, D. K. Fork, R. M. Wolf, and T. Shiosaki (Pittsburg, Pennsylvania: Materials Research Society), p. 91.

Yuan, J., Gu, E., Gester, M., Bland, J. A. C., and Brown, L. M., 1994, J. appl. Phys., 75, 6501.

Zener, C., 1951, Phys. Rev., 82, 403.

Zhang, J., Cui, G. J., Gordon, D., Van Buskirs, P., and Steinbeck, J., 1993, Ferroelectric Thin Films III, Materials Research Society Symposium Proceedings, Vol. 310, edited by E. R. Myers, B. A. Tuttle, S. B. Desu and P. K. Larsen (Pittsburg, Pennsylvania: Materials Research Society), p. 249.

Zhang, J., Gardiner, R. A., Kirlin, P. S., Boerstler, R. W., and Steinbeck, J., 1992, Appl. Phys. Lett., 61, 2884. 